\title{
A UNIQUENESS THEOREM FOR HAAR AND WALSH SERIES $\left({ }^{1}\right)$
}

\author{
BY \\ WILLIAM R. WADE
}

1. Introduction. It is well known that if a trigonometric series converges to an integrable function, except possibly in a countable set, and the series' coefficients converge to zero then that series is the Fourier series of the limit function [8, p. 329].

This problem for Walsh series was open for many years, but in 1965 two independent solutions were published: [2] and [3]. By combining the American and Soviet techniques we are able to obtain a theorem which contains the Walsh series result in [2] but which has a form similar to the theorem in [3]. Following the Soviet example, we will prove the result for Haar series and obtain the Walsh series result as a corollary.

In this paper $E$ will represent a countable subset of [0,1], and D.R. will represent the set of dyadic rationals. Given a Borel set $A, I_{A}(x)$ will denote the characteristic function of the set $A$.

The Haar system $\left\{\chi_{k}\right\}_{k=0}^{\infty}$ is defined as $\chi_{0}(x)=1, \chi_{1}(x)=I_{[0,1 / 2)}(x)-I_{(1 / 2,1]}(x)$; in general writing $k^{\prime}=2^{n}+k, 0 \leqq k<2^{n}$ where $n$ is the largest power of 2 which is less than or equal to $k^{\prime}$, we define

$$
\begin{aligned}
& \chi_{k}(x)=\chi_{n}^{(k)}(x)=\left(2^{n}\right)^{1 / 2} \quad 2 k-2 / 2^{n+1}<x<2 k-1 / 2^{n+1}, \\
& =-\left(2^{n}\right)^{1 / 2} \quad 2 k-1 / 2^{n+1}<x<2 k / 2^{n+1}, \\
& =\left(2^{n} / 4\right)^{1 / 2} \quad x=k-1 / 2^{n}, \\
& =-\left(2^{n} / 4\right)^{1 / 2} \quad x=k / 2^{n} \text {, } \\
& =0 \quad \text { otherwise. }
\end{aligned}
$$

We will denote

$$
\left(2 k-2 / 2^{n+1}, 2 k-1 / 2^{n+1}\right)=\Delta_{k^{\prime}}^{(1)}, \quad\left(2 k-1 / 2^{n+1}, 2 k / 2^{n+1}\right)=\Delta_{k^{\prime}}^{(2)}
$$

and these two open intervals will be referred to as the positive (respectively negative) support of the $k$ th Haar function. Alexits [1] proves this sequence is a complete orthonormal system.

We define the Walsh system $\left\{\Psi_{k}\right\}_{k=0}^{\infty}$ by letting $\Phi_{0}(x)=I_{[0,1 / 2)}(x)-I_{[1 ; 2,1]}(x)$, $\Phi_{n}(x)=\Phi_{0}\left(2^{n} x\right)$ (where $\Phi_{0}$ is extended by periodicity of period 1) and then defining $\Psi_{0}(x)=1, \Psi_{n}(x)=\Phi_{n_{1}}(x) \cdots \Phi_{n_{r}}(x)$ where $n=\sum_{i=1}^{r} 2^{n_{i}}$ and the $n_{i}$ are uniquely

Received by the editors October $1,1968$.

(1) This research was done in partial satisfaction for the Doctor of Philosophy degree at the University of California, Riverside, under the direction of Professor Victor L. Shapiro. 
determined by $n_{i+1}<n_{i}$. Walsh [7] proves this sequence is a complete orthonormal system.

Both the Walsh and Haar systems are extended by periodicity of period 1 to the whole real line. Kacmarzc noted [1, p. 62] that if $k^{\prime}=2^{n}+k, 0 \leqq k<2^{n}$ and $n$ largest such that $2^{n} \leqq k^{\prime}$, then

$$
\Psi_{k^{\prime}}(x)=\frac{1}{\left(2^{n}\right)^{1 / 2}} \sum_{j=1}^{2^{n}} \varepsilon_{k^{\prime} j} \chi_{n}^{(j)}(x) \text { for } x \notin \mathrm{D} . \mathrm{R} .
$$

where $\varepsilon_{j k^{\prime}}= \pm 1$ and the matrix [ $\left.\varepsilon_{j k^{\prime}}\right]$ has orthogonal rows. If $S$ is a Haar or Walsh series, $S_{n}$ will denote the partial sum of order $n-1$.

Given a Haar series $S(x)=\sum_{k=0}^{\infty} a_{k} \chi_{k}(x)$, we shall say $S$ satisfies condition $G$ if for every $x_{0} \in[0,1]$,

$$
\lim _{j \rightarrow \infty} \frac{a_{k_{j}}}{\chi_{k,}\left(x_{0}\right)}=0
$$

where $k_{j}$ are all those integers $p$ for which $\chi_{p}\left(x_{0}\right) \neq 0$. We note that this growth condition is essential to Theorem 1 since

$$
\chi_{0}(x)+\sum_{n=0}^{\infty}\left(2^{n}\right)^{1 / 2} \chi_{2^{n}}(x) \equiv 0 \quad \text { on }(0,1]
$$

but the series fails to satisfy (2) only at the point $x_{0}=0$.

We shall say a function $g$ is in the class $\mathscr{A}$ if there is a closed countable set $A_{g} \subseteq[0,1]$ such that $g$ is locally integrable in $(0,1) \sim A_{g}$. We note that $L^{1}[0,1]$ is a proper subset of $\mathscr{A}$ since $A_{\{1 / x\}}=\{0\}$.

We shall prove:

THEOREM 1. Let $S(x)=\sum_{k=0}^{\infty} a_{k} \chi_{k}(x)$ be a Haar series satisfying condition $G$ such that for some function $g \in \mathscr{A}$ and subsequence of natural numbers $\left\{n_{j}\right\}$

(i) $\lim _{j \rightarrow \infty} S_{2^{n}}(x)=g(x)$ in measure;

(ii) $\lim \sup _{j \rightarrow \infty}\left|S_{2^{n}}(x)\right|<\infty, x \notin E$;

(iii) $\lim \sup _{j \rightarrow \infty} S_{2^{n_{j}}}(x)$ dominates an integrable function $f(x)$ for $x \notin E$.

Then $g$ is integrable and $S$ is the Haar Fourier series of $g$.

THEOREM 2. Let $S(x)=\sum_{k=0}^{\infty} a_{k} \Psi_{k}(x)$ be a Walsh series such that for a function $g \in \mathscr{A}$ and some subsequence of natural numbers $\left\{n_{j}\right\}$

(i) $\lim _{j \rightarrow \infty} S_{2^{n}}(x)=g(x)$ in measure;

(ii) $\lim \sup _{j \rightarrow \infty}\left|S_{2^{n}}(x)\right|<\infty$ for $x \notin E$;

(iii) $\lim \sup _{j \rightarrow \infty} S_{2^{n}}(x)$ dominates an integrable function $f(x)$ for $x \notin E$;

(iv) $\lim _{k \rightarrow \infty} a_{k}=0$.

Then $g$ is integrable and $S$ is the Walsh Fourier series of $g$.

2. Fundamental lemmas. By $\alpha_{n}=\alpha_{n}(x)$ and $\beta_{n}(x)=\beta_{n}$ we shall mean

$$
\alpha_{n}=P \cdot 2^{-n} \leqq x<(P+1) 2^{-n}=\beta_{n}
$$


and $\alpha_{n}^{\prime}(x)=\alpha_{n}(x)$ if $x \notin$ D.R., $\alpha_{n}^{\prime}(x)=\alpha_{n}(x)-2^{-n}$ otherwise. Given a Haar series $S(x)=\sum_{k=0}^{\infty} a_{k} \chi_{k}(x)$ we define

$$
L\left(S, x, n_{j}\right)=\lim _{j \rightarrow \infty} \sum_{k=0}^{2^{n_{j}-1}} a_{k} \int_{0}^{x} \chi_{k}(u) d u=\lim _{j \rightarrow \infty} L_{2^{n_{j}}}(S, x)
$$

when this limit exists. In case $L(S, x, n)$ exists we will write it as $L(S, x)$ following the notation in [5].

LEMMA 1. If $S(x)=\sum_{k=0}^{\infty} a_{k} \chi_{k}(x)$ is a Haar series satisfying condition $G$, then for every $x$ and $n \geqq 0$ both $L\left(S, \alpha_{n}(x)\right)$ and $L\left(S, \beta_{n}(x)\right)$ exist and are finite. Furthermore

$$
\begin{aligned}
& L\left(S, \beta_{n}(x)\right)-L\left(S, \alpha_{n}(x)\right)=2^{-n} S_{2^{n}}(x), \\
& L\left(S, \beta_{n}(x)\right)-L\left(S, \alpha_{n}(x)\right)=o(1) \text { as } n \rightarrow \infty .
\end{aligned}
$$

Proof. We use [3, Lemma 1] as an outline of the proof of this lemma. To show (6), we use (5) and the fact that condition $G$ implies $2^{-n} S_{2^{n}}(x)=o(1)$.

To see this, given $\varepsilon>0$ and $x_{0} \in[0,1]$ we choose $N$ sufficiently large so that $j \geqq N$ implies

$$
\left|a_{k_{j}}\right|<\varepsilon \cdot\left|\chi_{k_{j}}\left(x_{0}\right)\right|
$$

where $k_{j}$ are defined in the definition of condition $G$. We then recall from the very definition of the Haar functions that the set

$$
\left\{\chi_{k}^{(1)}\left(x_{0}\right), \chi_{k}^{(2)}\left(x_{0}\right), \ldots, \chi_{k}^{\left(2^{k}\right)}\left(x_{0}\right)\right\}
$$

has at most two nonzero elements for each $k$, and that $\left|\chi_{k}^{(p)}\left(x_{0}\right)\right|^{2} \leqq 2^{k}$.

Combining (7) and (8) if $n>N$,

$$
\left|\frac{S_{2^{n}}\left(x_{0}\right)}{2^{n}}\right| \leqq \sup _{0 \leqq j \leqq N} \frac{\left|a_{k_{j}}\right| \cdot N}{\left(2^{n}\right)^{1 / 2}}+2 \varepsilon \cdot \frac{\sum_{k=0}^{n} 2^{k}}{2^{n}} .
$$

Hence taking lim sup of (9) as $n \rightarrow \infty$ we obtain $S_{2^{n}}\left(x_{0}\right)=o\left(2^{n}\right)$.

The following lemma is the Haar series analogue of a lemma in [3], and the proof is essentially the same.

LEMMA 2. If $S(x)=\sum_{k=0}^{\infty} a_{k} \chi_{k}(x)$ is a Haar series satisfying condition $G$ and if $L\left(S, x, n_{j}\right)$ exists and is finite, then

$$
\begin{aligned}
& L\left(S, \alpha_{n_{j}}^{\prime}\right) \rightarrow L\left(S, x, n_{j}\right) \text { as } j \rightarrow \infty, \\
& L\left(S, \beta_{n_{1}}\right) \rightarrow L\left(S, x, n_{j}\right) \text { as } j \rightarrow \infty .
\end{aligned}
$$

The following lemma is proved in a similar manner to Lemma 3 of [3].

LEMMA 3. Let $G(x)$ be defined in $a<x<b$ and satisfy:

(i) Except perhaps on a countable set $Z$, $\lim _{\inf _{j \rightarrow \infty}} 2^{n_{j}}\left[G\left(\beta_{n_{j}}\right)-G\left(\alpha_{n}\right)\right] \leqq 0$.

(ii) For all $x \in(a, b)$, both $G\left(\alpha_{n j}^{\prime}\right) \rightarrow G(x), G\left(\beta_{n_{j}}\right) \rightarrow G(x)$ as $j \rightarrow \infty$.

Then $G(x)$ is monotone nonincreasing in $(a, b)$. 
Lemma 4. Let $S(x)=\sum_{k=0}^{\infty} a_{k} \chi_{k}(x)$ be a Haar series satisfying condition $G$, let $f$ be a finite valued function, integrable over $(a-\varepsilon, b+\varepsilon)$ for $\varepsilon>0$, and let $\left\{n_{j}\right\}$ be a subsequence of the natural numbers. Suppose further that $Z$ is a countable subset of $(a-\varepsilon, b+\varepsilon)$ and that

(i) $L\left(S, x, n_{j}\right)$ exists and is finite for every $x \in(a-\varepsilon, b+\varepsilon)$;

(ii) $+\infty>\lim \sup _{j \rightarrow \infty} S_{2^{n}}(x) \geqq f(x)$ for $x \notin Z$.

Then

$$
\limsup _{j \rightarrow \infty} S_{2^{n_{j}}}(x)=\liminf _{j \rightarrow \infty} S_{2^{n}}(x) \quad \text { a.e. in }(a, b)
$$

and this function is integrable over $(a, b)$.

Proof. From hypothesis (ii) and (5) we have

$$
\limsup _{j \rightarrow \infty} 2^{n_{\jmath}}\left[L\left(S, \beta_{n_{\jmath}}\right)-L\left(S, \alpha_{n_{\jmath}}\right)\right] \geqq f(x) \text { for } x \in(a-\varepsilon, b+\varepsilon) \sim Z .
$$

Using the Vitali-Carathéodory Theorem [5, p. 75] we choose an upper semicontinuous function $f_{1} \leqq f$ in $L^{1}(a-\varepsilon, b+\varepsilon)$.

Let $F_{1}(x)=\int_{a-\varepsilon / 2}^{x} f_{1}(u) d u$ for $x \in(a-\varepsilon / 2, b+\varepsilon)$. Clearly since $\left|\alpha_{n_{j}}-\beta_{n_{j}}\right|=2^{-n_{j}}$,

$$
\liminf _{j \rightarrow \infty} 2^{n_{j}}\left[F_{1}\left(\beta_{n}\right)-F_{1}\left(\alpha_{n}\right)\right] \leqq f(x) \text { for } x \in(a-\varepsilon / 2, b+\varepsilon) \text {. }
$$

Thus by (13), for $x \in(a-\varepsilon / 2, b+\varepsilon) \sim Z$,

$$
\liminf _{j \rightarrow \infty} 2^{n_{j}}\left\{F_{1}\left(\beta_{n_{j}}\right)-L\left(S, \beta_{n_{j}}\right)-\left[F_{1}\left(\alpha_{n_{j}}\right)-L\left(S, \alpha_{n_{j}}\right)\right]\right\} \leqq 0 .
$$

Now Lemma 2 and continuity of $F_{1}$ imply hypothesis (ii) of Lemma 3 is also satisfied by the function $F_{1}(x)-L\left(S, x, n_{j}\right)$, so by Lemma 3 this function is monotone nonincreasing in $(a-\varepsilon / 2, b+\varepsilon)$.

Thus $L\left(S, x, n_{j}\right)$ has derivatives almost everywhere which are integrable on every closed subinterval of $(a-\varepsilon / 2, b+\varepsilon)$. Hence (12) is a consequence of the equality

$$
\frac{L\left(S, \beta_{n_{j}}\right)-L\left(S, \alpha_{n_{j}}\right)}{\beta_{n_{j}}-\alpha_{n_{j}}}=S_{2^{n_{j}}}(x) .
$$

Lemma 5. If $S^{\prime}(x)=\sum_{k=0}^{\infty} c_{k} \chi_{k}(x)$ is the Haar Fourier series of an integrable function $f(x)$ then

$$
\begin{gathered}
S^{\prime} \text { satisfies condition } G, \\
\lim _{n \rightarrow \infty} \int_{0}^{1}\left|S_{n}^{\prime}(x)-f(x)\right| d x=0 .
\end{gathered}
$$

Proof. By assumption $c_{k}=\int_{0}^{1} f(x) \chi_{k}(x) d x$. Thus

$$
\left|c_{k}\right| \leqq \max _{x}\left|\chi_{k}(x)\right|\left\{\int_{\Delta_{k}^{(1)} \cup \Delta_{k}^{(2)}}|f(x)| d x\right\}
$$

But $f$ is integrable, so $m\left(\Delta_{k}^{(1)} \cup \Delta_{k}^{(2)}\right) \rightarrow 0$ implies

$$
\frac{\left|c_{k}\right|}{\max _{x}\left|\chi_{k}(x)\right|} \rightarrow 0 \quad \text { as } k \rightarrow \infty
$$


But if $\chi_{k}\left(x_{0}\right) \neq 0$ then $\left|\chi_{k}\left(x_{0}\right)\right| \geqq \frac{1}{2} \max _{x}\left|\chi_{k}(x)\right|$ so (16) is proved. (17) is the theorem appearing in [6].

We quote the main lemma of [2].

LemMA 6. Suppose $S(x)=\sum_{k=0}^{\infty} a_{k} \chi_{k}(x)$ is a Haar series satisfying condition $G$ and $f$ is an integrable function whose Haar Fourier series is $S^{\prime}(x)=\sum_{k=0}^{\infty} c_{k} \chi_{k}(x)$. Suppose also that for some subsequence of natural numbers $\left\{n_{j}\right\}, S_{2^{n}}(x)$ converges to $f(x)$ in measure.

Let $x_{0} \in[0,1]$ and $k_{0}$ be an integer such that

$(\alpha)$ inside $\Delta_{k_{0}}^{\left(i_{0}\right)}, i_{0}=1$ or $2, S_{k_{0}+1}(x), S_{k_{0}+1}^{\prime}(x)$ have different constant values,

( $\beta) k>k_{0}$ implies $\chi_{k}$ is nonzero either in $\Delta_{k_{0}}^{\left(i_{0}\right)}$ or outside it.

Then for any $M>0$ and any positive integer $N$ we can find an $n_{j}$, a natural number $p$ and an interval of the form $\Delta_{p}^{\left(i_{p}\right)}, i_{p}=1$ or 2 such that

(1) $2^{n_{j}}>N$,

(2) $x_{0} \notin \Delta_{p}^{\left(i_{p}\right)-}$ and $\Delta_{p}^{\left(i_{p}\right)-} \subset \Delta_{k_{0}}^{\left(i_{0}\right)}$,

(3) $\left|S_{2^{n j}}(x)\right|>M$ for $x \in \Delta_{p}^{\left(i_{p}\right)}$ and is constant there,

(4) $p, \Delta_{p}^{\left(i_{p}\right)}$ satisfy $(\alpha),(\beta)$.

We close this section by recalling a theorem of Vitali $[4$, p. 152].

LEMMA 7. If $\left\{u_{n}\right\}_{n=0}^{\infty}$ is a sequence of functions which have equiabsolutely continuous integrals; i.e., given $\varepsilon>0$ there is $a \delta$ such that for $E \subseteq[0,1], m(E)<\delta$ implies

$$
\left|\int_{E} u_{n}(x) d x\right|<\varepsilon \text { for all } n ;
$$

and if $u_{n}(x) \rightarrow f(x)$ in measure, then $f(x)$ is integrable and

$$
\lim _{n \rightarrow \infty} \int_{0}^{1} u_{n}(x) d x=\int_{0}^{1} f(x) d x
$$

3. Proof of Theorem 1. Since $g \in \mathscr{A}$, given $x_{0} \notin A_{g}$ we can find an interval of the form $\Delta_{j_{0}}^{(i)}$ containing $x_{0}$ such that $\Delta_{j_{0}}^{(i)} \cap A_{g}=\varnothing$, and $g$ is integrable over $\Delta_{j_{0}}^{(i)}$. We claim that $L\left(S, x_{0}, n_{j}\right)$ exists and is finite. By Lemma 1 , we may assume $x_{0}$ is not a dyadic rational.

Consider the function

$$
g^{*}(x)=\left[g(x)-S_{j_{0}+1}(x)\right] I_{\Delta_{j 0}^{(i)}}(x) .
$$

By choice of $j_{0}, g^{*}$ is integrable in $[0,1]$. By hypothesis (i), $S_{2^{n_{j}}}(x) I_{\Delta_{j_{0}}^{(i)}}(x) \rightarrow g(x)$ $\cdot I_{\Delta_{j 0}^{(1)}}(x)$ in measure, so if we define

$$
T(x)=\left[S(x)-S_{j_{0}+1}(x)\right] I_{\Delta_{j 0}^{(1)}}(x)
$$

we have by (18)

$$
\lim _{j \rightarrow \infty} T_{2^{n_{j}}}(x)=g^{*}(x) \text { in measure. }
$$


We note that $T$ is actually a Haar series, say $T(x)=\sum_{k=0}^{\infty} \alpha_{k} \chi_{k}(x)$, and since $\left|\alpha_{k}\right| \leqq\left|a_{k}\right|, T$ satisfies condition $G$. Let $S^{\prime}(x)=\sum_{k=0}^{\infty} c_{k} \chi_{k}(x)$ be the Haar Fourier series of $g^{*}$. We claim $S^{\prime}$ and $T$ are the same series.

Suppose not, and let $k_{0}$ be the least integer for which $\alpha_{k_{0}} \neq c_{k_{0}}$. Clearly $T_{k_{0}+1}(x)$ $-S_{k_{0}+1}^{\prime}(x) \equiv d$ in $\Delta_{k_{0}}^{\left(i_{0}\right)}$ where $d$ is a nonzero constant and $i_{0}=1$ or 2 .

Let $E \cup$ D.R. $=\left\{Z_{1}, Z_{2}, \ldots\right\}$; we use Lemma 6 countably many times to obtain sequences $\left\{n_{j_{k}}\right\}_{k=1}^{\infty},\left\{p_{k}\right\}_{k=1}^{\infty}$ such that

$$
\begin{aligned}
& Z_{k} \notin \Delta_{p_{k}}^{\left(i_{p_{k}}\right)-} \subset \Delta_{p_{k-1}}^{\left(i_{p_{k}-1}\right)} \text { for } k=2,3, \ldots, \\
& \quad\left|T_{2^{n_{k}}}(x)\right|>k \text { for } x \in \Delta_{p_{k}}^{\left(i_{p_{k}}\right)}, \quad k=1,2, \ldots
\end{aligned}
$$

Since the dyadic rationals are excluded from the nested sequence $\Delta_{p_{k}}^{\left(i_{p_{k}}\right)}, m\left(\Delta_{p_{k}}^{\left(i_{p_{k}}\right)}\right)$ tends to zero as $k \rightarrow \infty$. By (21) we let $\xi \in \bigcap_{k=1}^{\infty} \Delta_{p_{k}}^{\left(i_{p_{k}}\right)}$. Then by (22),

$$
\limsup _{k \rightarrow \infty}\left|S_{2^{n} j_{k}}(\xi)\right|=\infty
$$

which implies by hypothesis (ii) that $\xi \in E \subseteq\left\{Z, Z_{2}, \ldots\right\}$ which contradicts (21). Thus the assumption was false and $S^{\prime} \equiv T$.

Thus by (19), if $n>j_{0}+1$ and $x \in \Delta_{j_{0}}^{(i)}$,

$$
S_{n}(x)=T_{n}(x)+S_{j_{0}+1}(x) .
$$

Since $T$ is a Fourier series and $S_{j_{0}+1}$ is a polynomial we use (17) and hypothesis (i) to see $S_{n}$ satisfies the hypotheses of Lemma 7 inside $\Delta_{j_{0}}^{(i)}$. Choose $\rho \in$ D.R. $\cap \Delta_{j_{0}}^{(i)}$ to the left of $x_{0}$, and use Lemma 7 to obtain

$$
\begin{aligned}
L\left(S, x_{0}, n_{j}\right)-L(S, \rho) & =\lim _{j \rightarrow \infty} \int_{\rho}^{x_{0}} S_{2^{n_{j}}}(u) d u \\
& =\int_{\rho}^{x_{0}} g(u) d u<\infty
\end{aligned}
$$

since $\left(\rho, x_{0}\right) \subseteq \Delta_{j_{0}}^{(i)}$. But by Lemma $1, L(S, \rho)$ is finite and thus $L\left(S, x_{0}, n_{j}\right)$ exists and is finite. The claim is thus true.

Let $N=\left\{x \in(0,1) \mid L\left(S, x, n_{j}\right)\right.$ does not exist $\}$. By our claim $N \subseteq A_{g}$. If we can show that $N$ has no isolated points, then $\bar{N} \subseteq\left(A_{g}\right)^{-}=A_{g}$ would be a perfect countable set, which would force $N$ to be empty. But by (4) and orthogonality of the Haar functions $L(S, 0)=0, L(S, 1)=a_{0}$, and so we will have shown that $L\left(S, x, n_{j}\right)$ exists and is finite everywhere in $[0,1]$.

Suppose indeed that $N$ has an isolated point $x_{0}$. Then for numbers $0<a<c$ $<d<b<1, L\left(S, x, n_{j}\right)$ exists and is finite in $\left[c, x_{0}\right) \cup\left(x_{0}, d\right]$. Choose $f_{1}$ by the Vitali-Carathéodory Theorem as in Lemma 4, and define $F_{1}(x)=\int_{0}^{x} f_{1}(u) d u$. Using (15), Lemma 2, hypothesis (ii), and continuity of $F_{1}$,

$$
\begin{gathered}
\liminf _{j \rightarrow \infty} 2^{n_{j}}\left\{F_{1}\left(\beta_{n_{j}}\right)-L\left(S, \beta_{n_{j}}\right)-\left[F\left(\alpha_{n_{j}}\right)-L\left(S, \alpha_{n_{j}}\right)\right]\right\} \leqq 0 \text { for } x \notin E, \\
F_{1}\left(\alpha_{n_{j}}^{\prime}\right)-L\left(S, \alpha_{n_{j}}^{\prime}\right) \rightarrow F_{1}(x)-L\left(S, x, n_{j}\right), \\
F_{1}\left(\beta_{n_{j}}\right)-L\left(S, \beta_{n_{j}}\right) \rightarrow F_{1}(x)-L\left(S, x, n_{j}\right) .
\end{gathered}
$$


Thus by Lemma $3, F_{1}(x)-L\left(S, x, n_{j}\right)$ is monotone nonincreasing in each of the intervals $\left(c, x_{0}\right),\left(x_{0}, d\right)$. But $F_{1}$ is continuous so $L\left(S, x, n_{j}\right)$ has a right and left limit (in the extended real plane) as $x \rightarrow x_{0}$. By (6) these limits must be equal, and by monotonicity they are finite. Thus by using (4), and the fact that the first $2^{n_{j}}$ Haar functions are constant in $\left(\alpha_{n g}, \beta_{n_{j}}\right)$

$$
\begin{aligned}
& \left|L_{2^{n_{j}}}\left(S, x_{0}\right)-L_{2^{n_{j}}}\left(S, \alpha_{n_{\jmath}}\right)\right|=\left|\int_{\alpha_{n_{j}}}^{x_{0}} S_{2^{n_{j}}}(u) d u\right| \\
& =\left|\left(x_{0}-\alpha_{n}\right) \cdot S_{2^{n},}\left(x_{0}\right)\right| \\
& \leqq \frac{\left|S_{2^{n_{j}}}\left(x_{0}\right)\right|}{2^{n_{j}}}=o(1)
\end{aligned}
$$

by (9). Thus $L_{2^{n_{j}}}\left(S, x_{0}\right)=L\left(S, \alpha_{n}\right)+o(1)$ which implies $L\left(S, x_{0}, n_{j}\right)$ exists and is finite, and we have proved $N$ has no isolated points.

By periodicity of the Haar functions we conclude that $L\left(S, x, n_{j}\right)$ exists and is finite for all real $x$. Hence using Lemma 4 we conclude $g \in L^{1}[-1 / 2,3 / 2]$ and $S_{2^{n_{j}}}(x)$ $\rightarrow g(x)$ almost everywhere.

We now proceed as we did locally for $g^{*}$. If the Haar Fourier series of the integrable function $g(x)$ is not identically equal to the series $S$, then using Lemma 6 countably many times we conclude the $E$ is uncountable contrary to hypothesis. Thus $S$ is the Haar Fourier series of the function $g$ and Theorem 1 is established.

4. Proof of Theorem 2. Using (1) and defining

$$
\alpha_{k}=\alpha_{n}^{\left(k^{\prime}\right)}=\sum_{i=2^{n}}^{2^{n+1}-1} \frac{\varepsilon_{i k}}{\left(2^{n}\right)^{1 / 2}} a_{i}
$$

we see that the Haar series

satisfies

$$
T(x)=\sum_{k=0}^{\infty} \alpha_{k} \chi_{k}(x)
$$

$$
T_{2^{n}}(x)=S_{2^{n}}(x) \text { for } x \notin \mathrm{D} . \mathrm{R} .
$$

Thus the Haar series $T$ satisfies hypotheses (i), (ii) and (iii) of Theorem 1, for the countable set $E \cup$ D.R. We now claim that hypothesis (iv) implies $T$ satisfies condition $G$. To see this use (26) to obtain

$$
\left|\alpha_{n}^{(k)}\right| \leqq\left(2^{n}\right)^{1 / 2} \max _{2^{n} \leqq i<2^{n+1}}\left|a_{i}\right| .
$$

But by definition of the Haar functions, $\max _{x}\left|\chi_{n}^{(k)}(x)\right|=\left(2^{n}\right)^{1 / 2}$, so we use the argument in Lemma 5 to conclude $T$ satisfies condition $G$.

Thus by Theorem $1, g$ is integrable, and $T$ is its Haar Fourier series. We now observe that (26) implies

$$
a_{k}=\sum_{i=2^{n}}^{2^{n+1}-1} \frac{\varepsilon_{i k}}{\left(2^{n}\right)^{1 / 2}} \alpha_{i}
$$


Hence by (1), (28) and Theorem 1,

$$
\begin{aligned}
a_{k} & =\sum_{i=2^{n}}^{2^{n+1}-1} \frac{\varepsilon_{i k}}{\left(2^{n}\right)^{1 / 2}} \int_{0}^{1} g(x) \chi_{i}(x) d x \\
& =\int_{0}^{1} g(x) \sum_{i=2^{n}}^{2^{n+1}-1} \frac{\varepsilon_{i k} \chi_{i}(x)}{\left(2^{n}\right)^{1 / 2}} d x \\
& =\int_{0}^{1} g(x) \Psi_{k}(x) d x
\end{aligned}
$$

which means that $S$ is the Walsh Fourier series of the integrable function $g$.

\section{REFERENCES}

1. G. Alexits, Convergence problems of orthogonal functions, transl. by I. Földes, Pergamon Press, New York, 1961.

2. F. G. Arutunjan and A. A. Talaljan, On uniqueness of Haar and Walsh series, Izv. Akad. Nauk SSSR 28 (1964), 1391-1408. (Russian)

3. R. B. Crittenden and V. L. Shapiro, Sets of uniqueness on the group $2^{\omega}$, Ann. of Math. 81 (1965), 550-564.

4. I. N. Natanson, Theory of functions of a real variable, transl. by L. F. Boron, Ungar, New York, 1955.

5. S. Saks, Theory of the integral, Hafner, New York, 1937.

6. J. Schauder, Eine Eigenschaft des Haarshen Orthogonalsystems, Math.Z.28(1928), 317-320.

7. J. L. Walsh, A closed set of normal orthogonal functions, Amer. J. Math. 55 (1923), 5-24.

8. A. Zygmund, Trigonometric series, Vol. I, Cambridge Univ. Press, Cambridge, 1959.

UNIVERSITY OF TENNESSEE,

KNOXVILle, TENNESSEE

UNIVERSITY OF CALIFORNIA,

Riverside, California 